\title{
Analysis of BMSCs-intervened viscoelasticity of sciatic nerve in rats with chronic alcoholic intoxication ${ }^{1}$
}

\author{
Chengdong Piao', Zhengwei Li", Jie Ding'I', Daliang Kong ${ }^{\mathrm{IV}}$
}

'PhD, Department of Orthopaedics, Second Hospital of Jilin University, China. Conception and design of the study. "PhD, Department of Orthopaedics, Second Hospital of Jilin University, China. Histopathological examinations.

"'Master, Department of Stomatology, Affiliated Hospital of Changchun University of Chinese Medicine, China. Acquisition of data.

IVPhD, Department of Orthopaedics, China-Japan Union Hospital, Jilin University, China. Technical procedures, analysis of data.

\begin{abstract}
Purpose: To investigate the impact of bone mesenchymal stem cells (BMSCs) intervention on the viscoelasticity of sciatic nerve in rats with chronic alcohol intoxication (CAI).

Methods: The CAI rat models were prepared, divided into model groups, and treated with either BMSCs or basic fibroblast growth factor (bFGF). Then the rats underwent electrophysiological test and the serum levels of malondialdehyde (MDA), superoxide dismutase (SOD), and metallothionein (MT) were measured. Histological observation, stress relaxation test, and creep test were performed for the sciatic nerve of the CAI model in each group.

Results: The MDA level of group BMSC was significantly lower $(p<0.05)$ than that of groups MOD (the CIA model) and BFGF. The SOD and MT levels were higher in group BMSC than in groups MOD and bFGF $(p<0.05)$. The motor nerve conduction velocity and amplitude were higher in group BMSC than in groups MOD and bFGF $(p<0.05)$. The amounts of 7200 s stress reduction and $7200 \mathrm{~s}$ strain increase of the sciatic nerve in group BMSC were greater than those in groups bFGF and MOD ( $p<0.05)$.

Conclusion: Bone mesenchymal stem cells can improve the metabolism of free radicals, restore the tissue morphology and viscoelasticity of the chronic alcohol intoxication animal model, and positively affect the repairing of the injured sciatic nerve.

Key words: Alcoholic Intoxication. Mesenchymal Stromal Cells. Serum. Sciatic Nerve. Rats.
\end{abstract}




\section{Introduction}

Long-term effects of alcohol abuse can cause direct damage to the brain and liver as well as nervous system damage, resulting in judgment decline, emotional numbness, insight weakening, family discord, interpersonal tension, and other social problems ${ }^{1-3}$. Chronic alcohol intoxication (CAI)-induced peripheral neuropathy was discovered earlier, but caught people's attention only towards the end of the 19 th century 4 . Many scholars have conducted research on drug therapies against $\mathrm{CAl}^{5,6}$. Monforte et al. ${ }^{7}$ showed that long-term intake of ethanol can cause changes in the cytoskeletal structure of peripheral nerves, and affect the transportation among the axons, thus affecting the functional integration of neurons. Tan et $a .^{8}$ believe that alcohol intoxication (AI) causes functional and structural damages in the peripheral nerves and results in peripheral neuropathy, which is also related to the changes in membrane fluidity as well as in membrane ion channels and oxygen free radical production.

In recent years, bone marrow mesenchymal stem cells (BMSCs) and basic fibroblast growth factor (bFGF) have been used to repair the central and peripheral nerve injury in animals. Dezawa et al. ${ }^{9}$ transplanted Schwann cells differentiated from BMSCs to the nerve endings and confirmed, for the first time, that BMSCs have the ability to promote nerve regeneration. Wang et al. ${ }^{10}$ found that BMSCs have a significant protective effect on the spinal anterior horn neurons. Aebischer et al. ${ }^{11}$ bridged sciatic nerve defects, $15 \mathrm{~mm}$ and 7 $\mathrm{mm}$ in length, in rats by treating with bFGF for 4 weeks. They found that the nerve regeneration was successful, which is consistent with that by the intervention of bFGF in the central nervous system. bFGF can promote the regeneration of peripheral nerves. However, previous studies $^{5-11}$ did not analyze the viscoelastic mechanical properties after transplantation. We hypothesized that BMSC transplantation would restore the viscoelastic properties of the sciatic nerve in CAl and repair sciatic nerve injury to a certain extent. Therefore, this study aimed to verify our hypothesis and the property of viscoelasticity for clinical rehabilitation of sciatic nerve injury.

\section{- Methods}

This study was carried out in strict accordance with the recommendations in the Guide for the Care and Use of Laboratory Animals of the National Institutes of Health. The animal use protocol has been reviewed and approved by the Institutional Animal Care and Use Committee (IACUC) of Jilin University.

Hundred male Wistar rats ( 6 months old; 295-310g) were purchased from the Changchun High-tech Experimental Animal Center (license SCXK (Kyrgyzstan) 2003-0004) and randomly divided into the normal control group (CON, $n=25$ ), the CAl model group (MOD, $n=25$ ), the bFGF intervention group ( $b F G F, n=25$ ), and the BMSC intervention group (BMSC, $n=25$ ). The rats were housed in separate cages ( 25 rats per cage) and could access feed and drink water freely.

\section{Establishment of CAl animal model}

For the establishment and identification of the CAl rat model, we referred to an established protocol ${ }^{12}$. Briefly, the rats were gavaged with liquor, produced by Changchun Huanglongyan Wine Co., Ltd. (Changchun, Jilin, China; Volume ratio $50 \%, 10 \mathrm{~g} /(\mathrm{kg} \bullet \mathrm{d})$, twice (12-h interval). After continuous gavage with liquor for 60 days, the rats showed dull hair, unstable temperament, loss of appetite, and slowness of movement. Some rats showed mild symptoms, such as dysphoria, apathy, and indifferent reaction and some of them exhibited severe symptoms, such as activity 
reduction, dull/drowsy body, significantly increased sleep time, body weight growth, and less eating than group CON, proving the successful establishment of the CAI rat model.

Electrophysiological test: Alcoholism in humans or animals always resulted in damage to the peripheral nerves. The electrophysiological test was used to detect the sciatic nerve injury, which was used as an indicator of the successful model of alcoholism as well as to define the effect of the intervention of BMSCs and bFGF. Seventy-four days after the establishment of the model, all the rats underwent electrophysiological tests by NIM-Neuro 2.0 Medtronic electromyography (Minneapolis, Minnesota, USA). The rats were anesthetized by intraperitoneal injection with $10 \%$ chloral hydrate $(400 \mathrm{mg} / \mathrm{kg})$, then the bilateral sciatic nerve trunks were exposed in prone position, the EMG core electrode needle was implanted in the intrafusal muscle of the rat soleus, the ground lead was clipped to the skin edge of the wound with an alligator clip. Two motor potentials were introduced at proximal anastomotic site sciatic tubercle (P) and distal anastomotic site sciatic nerve branch (D) by parallel stimulation electrodes (50 $\mathrm{mA})$. The action potential, amplitude, action potential amplitude and latency were displayed by the instrument. The distance between two stimulating electrodes is measured by the vernier caliper, which was inputted into the instrument to get the motor nerve conduction velocity (MNCV).

\section{Drug intervention}

On Day 60, the CAl rat model was intervened with BMSCs produced by Shanghai Yiyan Biotechnology Co., Ltd. (Shanghai, China). The BMSCs were passaged in accordance with the manufacturer's instructions. When the BMSCs reached $90 \%$ confluence, they were digested with $0.05 \%$ trypsin and passaged at $2 \times 10^{4}$ cells $/ \mathrm{cm}^{2}$. The cell culture conditions included: passage cycle of 5 days; 1:2 passage ratio; medium change in 3 days; incubation at $37^{\circ} \mathrm{C}$ in $5 \% \mathrm{CO}_{2} / 95 \%$ air. The $5^{\text {th }}$ generation of BMSCs (approximately $5 \times 10^{5}$ cells per $\mathrm{mL}$; cultured in a $\mathrm{CO}_{2}$-saturated humidity incubator at $37^{\circ} \mathrm{C}$ with a volume fraction of 0.05 ) was injected into each rat through the tail vein with a microsyringe. The rats in group bFGF were intramuscularly injected with bFGF produced by Xi'an Rongzhi Biotechnology Co., Ltd. (Xi'an, Shaanxi, China) at a dose of $12 \mu \mathrm{g} / \mathrm{kg}$. $\mathrm{d}$ for a total of 14 days. The rats in groups CON and MOD were not given any intervention.

\section{Specimen sampling}

On Day 74, which is Day 14 after drug intervention, 20 rats in each group were taken and blood sample was taken from the orbit. The sample was left undisturbed for sometime, centrifuged, and $6 \mathrm{~mL}$ of fresh blood was collected. This blood was left undisturbed for $15 \mathrm{~min}$ in the air and centrifuged at $2000 \mathrm{r} / \mathrm{niin}$ for 20 min (4-5R; Hengnuo instrumentation Co., Ltd., Changsha, Hunan, China). The supernatant was cryopreserved at $-40^{\circ} \mathrm{C}$ for future use; the rats were then fixed on the animal operating table, one midline incision was made along the left and right posterior femur, the skin and subcutaneous tissue were cut open, the semitendinosus and semimembrane muscles were separated, and the right sciatic nerve was exposed and dissociated. Sciatic nerve $(25 \mathrm{~mm})$ was then horizontally excised in the middle of the femur. In each group, 25 specimens were sampled, which were wrapped by saline-soaked cloth, placed in a saline bath, and stored at $4^{\circ} \mathrm{C}$ for future use.

Determination of serum SOD, MDA, and MT levels

Malondialdehyde (MDA), superoxide dismutase (SOD), and metallothionein (MT) are important biological indicators, which were 
used to define the effect of alcohol intoxication and then to find whether the intervention of BMSCs and bFGF can restore MDA, SOD, and $M T$ in rat serum. The superoxide dismutase (SOD) kit, malondialdehyde (MDA) kit, and MT kit were obtained from Nanjing Jiancheng Bioengineering Institute (Nanjing, Jiangsu, China). The serum SOD was measured by the purine oxidase method. Briefly, $10 \mu \mathrm{L}$ of $10 \%$ supernatant of each group was taken and the absorbance was detected at a wavelength of $550 \mathrm{~nm}$ using a UV-1700 UV-Vis spectrophotometer manufactured by Shanghai Analytical Instrument Factory (Shanghai, China) and the SOD value was calculated according to the calculation formula specified in the SOD kit and the result is represented as $\mathrm{U} / \mathrm{mg}$ prot. The MDA content was measured by the thiobarbituric acid method using a UV-1700 UVVis spectrophotometer. The absorbance of the supernatant was measured at a wavelength of $532 \mathrm{~nm}$, which was used to calculate the MDA content according to the calculation formula specified in the MDA kit. The MDA content is represented as $\mathrm{nmol} / \mathrm{mg}$ prot. The serum MT test was performed according to the MT kit instructions, using the thiobarbituric acid colorimetric method.

\section{Stress relaxation assay of rat sciatic nerve}

Stress relaxation assay is an important biomechanical experiment. The stress relaxation assay of rat sciatic nerve is used to determine whether alcoholism has any effect on the stress relaxation characteristics of rats and whether the intervention of BMSCs and bFGF can restore it.

The experimental equipment was Model 55100 automatic control universal testing machine produced by the Changchun Research Institute for Testing Machines (Changchun, Jilin, China). The instrument used for measuring the length and diameter of the sciatic nerve sample was CGA-5 reading microscope produced by Changchun Third Optical Instrument Factory (Changchun, Jilin, China). The sample was $15 \mathrm{~mm}$ in length and $0.99-1.03 \mathrm{~mm}$ in diameter. Each sciatic nerve sample was preconditioned according to previous protocols ${ }^{13-16}$. The experimental temperature was set to $36.5 \pm 1^{\circ} \mathrm{C}$. Each sample was clamped in the fixture of the tester, and strain was applied to the specimen at an experimental speed of $50 \% / \mathrm{min}$. When the stress of each sciatic nerve specimen reached $0.5 \mathrm{MPa}$, the strain was kept constant while the stress continuously decreased with time changing. The experimental time was set to $120 \mathrm{~min}$.

\section{Sciatic nerve creep test}

The sciatic nerve creep test is an important biomechanics method, which can be used to find the effect of alcoholism on rat sciatic nerve and whether the intervention of BMSCs and bFGF can restore it.

The data acquisition, experimental temperature, specimen mounting method, and sample preconditioning of sciatic nerve creep test were the same as those in sciatic nerve stress relaxation test. The experimental speed was set as $0.5 \mathrm{GPa} / \mathrm{min}$ to perform the creep test. When the stress of the sciatic nerve reached $0.50 \mathrm{MPa}$, the stress was kept constant while the strain increased continuously with time. The experimental time was set to 120 $\min$.

\section{Observation of cross-sectional microstructure of sciatic nerve}

Histological results showed the injury of the sciatic nerve directly. Based on the morphology of the sciatic nerve, we can judge whether alcoholism can damage the sciatic nerve in rats and whether the intervention of BMSCs and bFGF can restore it.

One sciatic nerve was sampled from 
each group, followed by freezing, fixation with $40 \mathrm{~g} / \mathrm{L}$ paraformaldehyde, and sectioning. Hematoxylin was added for 3 min for staining, followed by color separation with ethanol-HCl (v/v=99:1), alkaline water blue staining, and $60 \mathrm{sec}$ eosin staining; each staining step was followed by specimen washing in tap water. The specimen was dehydrated with a gradient of ethanol for $5 \min (70 \%, 85 \%$, and $100 \%$, respectively), followed by hyalinization (10 min with xylene I and 10 min with xylene II), and final sealing in neutral resin. The cross section of the sciatic nerve was observed under an Olympus BX51 optical microscope (Tokyo, Japan) to understand the changes in the axons, nerve cells, myelin, and basement membrane.

\section{Statistical analysis}

The data are expressed as mean \pm standard deviation (SD) and analyzed using SPSS 16.0 software package (SPSS, Chicago, IL, USA). The difference in data between groups was analyzed using one-way ANOVA and Scheffe's method. $\mathrm{P}<0.05$ was regarded as statistically significant difference. The formula of stress-strain functional relation of each group was established by mathematical regression analysis.

\section{- Results}

Sciatic nerve electrophysiology

The electrophysiological results showed that the motor nerve conduction velocity (MNCV) and amplitude of group BMSC were higher than those of groups MOD and bFGF $(p<0.05)$. It means that alcoholism caused sciatic nerve injury in rats, and the intervention of BMSCs and bFGF helped recover from such injury (Table 1).

Table 1 - Electrophysiological results of sciatic nerve $(\bar{x} \pm \mathrm{SD})$.

\begin{tabular}{lll} 
Group & $\begin{array}{l}\text { Amplitude of action } \\
\text { potential }(\mathrm{mV})\end{array}$ & $\begin{array}{l}\text { MNCV } \\
(\mathrm{m} / \mathrm{s})\end{array}$ \\
\hline CON & $26.2 \pm 2.0$ & $49.1 \pm 2.7$ \\
BMSCs & $24.8 \pm 2.6^{\mathrm{a}}$ & $47.9 \pm 2.2^{\mathrm{a}}$ \\
bFGF & $23.2 \pm 2.3^{\mathrm{b}}$ & $45.9 \pm 2.6^{\mathrm{b}}$ \\
MOD & $21.1 .9 \pm 1.4^{\mathrm{c}}$ & $43.1 \pm 1.9^{\mathrm{c}}$ \\
\hline
\end{tabular}

Note: ${ }^{a} \mathrm{P}<0.05$, vs. CON, ${ }^{b} \mathrm{P}<0.05$, vs. BMSCs, ${ }^{\mathrm{c} P}<0.05$, vs. bFGF.

Levels of serum MDA, SOD, and MT

The serum levels of MDA, SOD, and MT are given in Table 2. Comparison of the levels of serum MDA, SOD, and MT among different groups showed that the contents of SOD and MT in group MOD were significantly lower than those in groups CON, bFGF, and BMSC $(p<0.05)$, and were significantly lower in group BMSC than in groups MOD and bFGF $(p<0.05)$.

It means that alcoholism affected the levels of serum MDA, SOD, and MT in rats, and the intervention of BMSCs and bFGF helped to recover these levels.

Table 2 - Results of MDA, SOD and MT ( $\bar{x} \pm S D)$.

\begin{tabular}{llll}
\hline Group & MDA(mmol $/ \mathrm{mL})$ & $\mathrm{MT}(\mathrm{mmol} / \mathrm{mL})$ & $\mathrm{SOD}(\mathrm{mmol} / \mathrm{mL})$ \\
\hline Con $(\mathrm{N}=15)$ & $3.21 \pm 0.62$ & $3.92 \pm 0.36$ & $56.9 \pm 10.1$ \\
BMSCs $(\mathrm{N}=15)$ & $3.62 \pm 0.64^{\mathrm{a}}$ & $3.41 \pm 0.41^{\mathrm{a}}$ & $49.3 . \pm 8.6^{\mathrm{a}}$ \\
bFGF $(\mathrm{N}=15)$ & $3.98 \pm 0.59^{\mathrm{b}}$ & $3.13 \pm 0.3^{\mathrm{b}}$ & $47.1 . \pm 7.8^{\mathrm{b}}$ \\
MOD $(\mathrm{N}=15)$ & $5.28 \pm 1.1^{\mathrm{c}}$ & $2.19 \pm 0.25^{\mathrm{c}}$ & $40.28 \pm 11.3^{\mathrm{c}}$ \\
\hline
\end{tabular}

Note: ${ }^{a} \mathrm{P}<0.05$, vs. CON; ${ }^{\mathrm{b}} \mathrm{P}<0.05$ vs. BMSCs; ${ }^{\mathrm{C}}<<0.05$, vs. bFGF. 
Stress relaxation of the sciatic nerve

The results of stress relaxation analysis showed that the decrease in stress at 7200s (120 min) in group BMSC was significantly higher than that in groups bFGF and MOD $(p<0.05$, Table 3$)$. The stress relaxation curves of the sciatic nerve are shown in Figure 1. It was found that alcoholism affected the stress relaxation of the sciatic nerve in rats, which was recovered after intervention with BMSCs and bFGF.

Table 3 - Stress relaxation of sciatic nerve $(\bar{x} \pm S D)$.

\begin{tabular}{ccccccccccccc}
\hline $\begin{array}{c}\sigma(\mathrm{MPa}) \\
\text { Groups }\end{array}$ & $0 \mathrm{~s}$ & $300 \mathrm{~s}$ & $600 \mathrm{~s}$ & $900 \mathrm{~s}$ & $1200 \mathrm{~s}$ & $1800 \mathrm{~s}$ & $\mathbf{2 4 0 0 s}$ & $3600 \mathrm{~s}$ & $\mathbf{4 8 0 0 s}$ & $5400 \mathrm{~s}$ & $6600 \mathrm{~s}$ & 7200s \\
\hline Con & 0.501 & 0.420 & 0.401 & 0.362 & 0.331 & 0.318 & 0.317 & 0.316 & 0.315 & 0.314 & 0.313 & 0.312 \\
& \pm 0.01 & \pm 0.02 & \pm 0.02 & \pm 0.02 & \pm 0.01 & \pm 0.02 & \pm 0.02 & \pm 0.02 & \pm 0.04 & \pm 0.02 & \pm 0.02 & \pm 0.02 \\
BMSCs & 0.501 & 0.431 & 0.412 & 0.373 & 0.352 & 0.338 & 0.337 & 0.336 & 0.335 & 0.334 & 0.333 & 0.332 \\
& $\pm 0.02^{\mathrm{a}}$ & $\pm 0.04^{\mathrm{a}}$ & $\pm 0.04^{\mathrm{a}}$ & $\pm 0.02^{\mathrm{a}}$ & $\pm 0.04^{\mathrm{a}}$ & $\pm 0.03^{\mathrm{a}}$ & $\pm 0.03^{\mathrm{a}}$ & $\pm 0.03^{\mathrm{a}}$ & $\pm 0.03^{\mathrm{a}}$ & $\pm 0.04^{\mathrm{a}}$ & $\pm 0.03^{\mathrm{a}}$ & $\pm 0.04^{\mathrm{a}}$ \\
bFGF & 0.501 & 0.442 & 0.431 & 0.383 & 0.358 & 0.357 & 0.356 & 0.355 & 0.354 & 0.353 & 0.352 & 0.351 \\
& $\pm 0.01^{\mathrm{b}}$ & $\pm 0.03^{\mathrm{b}}$ & $\pm 0.03^{\mathrm{b}}$ & $\pm 0.03^{\mathrm{b}}$ & $\pm 0.03^{\mathrm{b}}$ & $\pm 0.01^{\mathrm{b}}$ & $\pm 0.04^{\mathrm{b}}$ & $\pm 0.02^{\mathrm{b}}$ & $\pm 0.03^{\mathrm{b}}$ & $\pm 0.04^{\mathrm{b}}$ & $\pm 0.02^{\mathrm{b}}$ & $\pm 0.03^{\mathrm{b}}$ \\
MOD & 0.501 & 0.461 & 0.422 & 0.401 & 0.388 & 0.387 & 0.386 & 0.385 & 0.384 & 0.383 & 0.382 & 0.381 \\
& $\pm 0.01^{\mathrm{c}}$ & $\pm 0.03^{\mathrm{c}}$ & $\pm 0.03^{\mathrm{c}}$ & $\pm 0.03^{\mathrm{c}}$ & $\pm 0.02^{\mathrm{c}}$ & $\pm 0.03^{\mathrm{c}}$ & $\pm 0.04^{\mathrm{c}}$ & $\pm 0.03^{\mathrm{c}}$ & $\pm 0.02^{\mathrm{c}}$ & $\pm 0.03^{\mathrm{c}}$ & $\pm 0.04^{\mathrm{c}}$ & $\pm 0.03^{\mathrm{c}}$ \\
\hline
\end{tabular}

Note: ${ }^{a} \mathrm{P}<0.05$, vs. CON; ${ }^{\mathrm{b}} \mathrm{P}<0.05$ vs. BMSCs; ${ }^{\mathrm{C}} \mathrm{P}<0.05$, vs. bFGF.

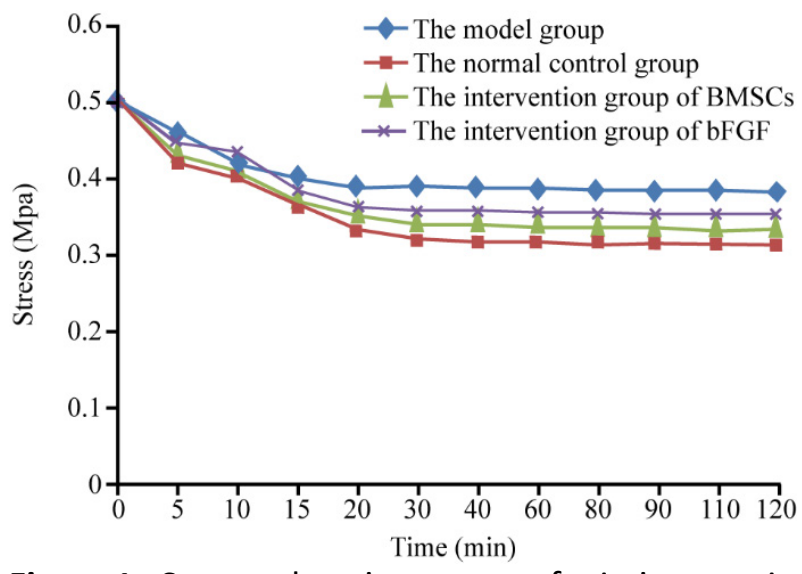

Figure 1 - Stress relaxation curves of sciatic nerve in different groups.

\section{Creep characteristic of the sciatic nerve}

The results of the creep test showed that the strain increase up to $7200 \mathrm{~s}$ in group BMSC was significantly greater than that in groups MOD and bFGF ( $p<0.05)$. It was found that alcoholism had a certain effect on the creep characteristic of the rat sciatic nerve, and the intervention of BMSCs and bFGF helped recover this property (Table 4). The creep curves of the sciatic nerve samples of each group are represented in Figure 2.

Table 4 - Test results of sciatic nerve creep $(\bar{x} \pm$ SD).

\begin{tabular}{cccccccccccccc}
$\begin{array}{l}\varepsilon(\%) \\
\text { Groups }\end{array}$ & 0s & $300 \mathrm{~s}$ & $600 \mathrm{~s}$ & $900 \mathrm{~s}$ & $1200 \mathrm{~s}$ & $1800 \mathrm{~s}$ & $2400 \mathrm{~s}$ & $3600 \mathrm{~s}$ & $4800 \mathrm{~s}$ & $\mathbf{5 4 0 0 s}$ & $6600 \mathrm{~s}$ & $7200 \mathrm{~s}$ \\
\hline Con & 29.21 & 31.23 & 32.31 & 33.90 & 34.81 & 35.06 & 35.07 & 35.08 & 35.09 & 35.10 & 35.11 & 35.12 \\
& \pm 1.6 & \pm 2.1 & \pm 2.0 & \pm 1.4 & \pm 2.2 & \pm 2.2 & \pm 2.3 & \pm 1.9 & \pm 2.6 & \pm 1.6 & $\pm 2.2^{2}$ & \pm 1.7 \\
BMSCs & 28.13 & 29.24 & 30.34 & 32.61 & 33.17 & 33.18 & 33.19 & 33.20 & 33.21 & 33.22 & 33.23 & 33.24 \\
& $\pm 1.4^{\mathrm{a}}$ & $\pm 1.6^{\mathrm{a}}$ & $\pm 1.4^{\mathrm{a}}$ & $\pm 1.7^{\mathrm{a}}$ & $\pm 1.3^{\mathrm{a}}$ & $\pm 2.6^{\mathrm{a}}$ & $\pm 1.6^{\mathrm{a}}$ & $\pm 2.0^{\mathrm{a}}$ & $\pm 1.8^{\mathrm{a}}$ & $\pm 2.5^{\mathrm{a}}$ & $\pm 1.8^{\mathrm{a}}$ & $\pm 1.9^{\mathrm{a}}$ \\
bFGF & 27.21 & 28.31 & 29.21 & 30.82 & 31.46 & 31.47 & 31.48 & 31.49 & 31.50 & 31.51 & 31.52 & 31.53 \\
& $\pm 0.9^{\mathrm{b}}$ & $\pm 1.8^{\mathrm{b}}$ & $\pm 0.8^{\mathrm{b}}$ & $\pm 1.3^{\mathrm{b}}$ & $\pm 1.8^{\mathrm{b}}$ & $\pm 2.1^{\mathrm{b}}$ & $\pm 2.4^{\mathrm{b}}$ & $\pm 1.8^{\mathrm{b}}$ & $\pm 2.1^{\mathrm{b}}$ & $\pm 2.2^{\mathrm{b}}$ & $\pm 2.4^{\mathrm{b}}$ & $\pm 2.2^{\mathrm{b}}$ \\
MOD & 25.82 & 26.21 & 27.32 & 28.12 & 29.13 & 29.65 & 29.66 & 29.67 & 29.68 & 29.69 & 29.70 & 29.71 \\
& $\pm 1.2^{\mathrm{c}}$ & $\pm 1.4^{\mathrm{c}}$ & $\pm 1.7^{\mathrm{c}}$ & $\pm 1.2^{\mathrm{c}}$ & $\pm 1.1^{\mathrm{c}}$ & $\pm 1.4^{\mathrm{c}}$ & $\pm 1.8^{\mathrm{c}}$ & $\pm 1.7^{\mathrm{c}}$ & $\pm 1.3^{\mathrm{c}}$ & $\pm 1.8^{\mathrm{c}}$ & $\pm 1.0^{\mathrm{c}}$ & $\pm 2.1^{\mathrm{c}}$ \\
\hline
\end{tabular}

Note: ${ }^{\mathrm{a}}<0.05$, vs. CON; ${ }^{\mathrm{b}}<<0.05$ vs. BMSCs; ${ }^{\mathrm{c} P}<0.05$, vs. bFGF. 


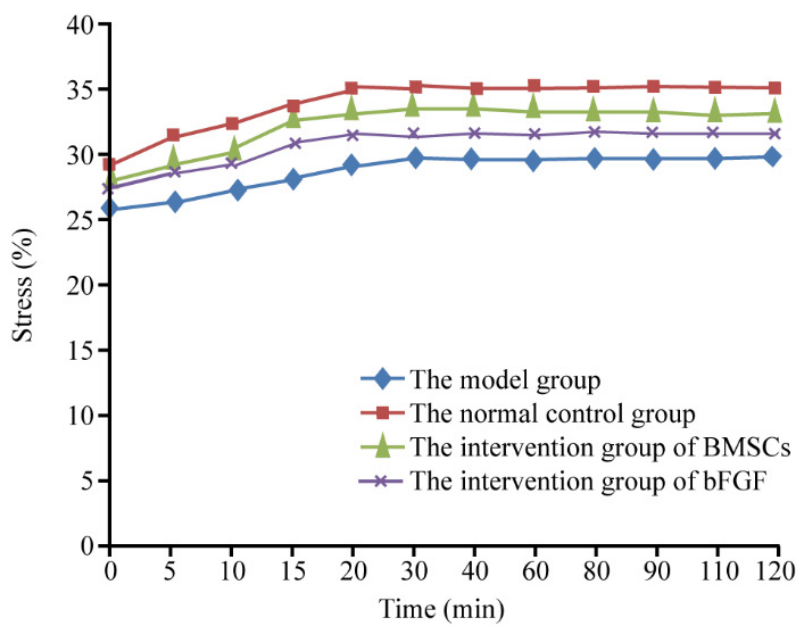

Figure $\mathbf{2}$ - Creep curves of sciatic nerve in different groups.

\section{Sciatic nerve morphology}

The results of optical microscopic observation of the sciatic nerve are shown in Figure 3. The observation revealed that the sciatic nerve fibers in group CON were arranged neatly, and the axons were surrounded by the peripheral myelin sheaths. The axons and other morphologies are clearly shown in Figure 3A. The nerve fibers in group MOD were not arranged neatly and exhibited axonal swelling, nerve fiber thickness variation, myelin segmental loss, and axon atrophy or even disappearance; the dorsal root ganglion neurons exhibited edema and were disorderly arranged; and the intercellular structure was loose (Figure 3B). Most of the sciatic nerve fibers in group BMSC were regular, with a small number exhibiting myelin sheath loss, nerve fiber swelling, and lighter axon atrophy; a small number of neurons in the dorsal root ganglion were loosely arranged, and the intercellular structure was slightly loose (Figure $3 \mathrm{C}$ ). The sciatic nerve structure in group bFGF was more complete, with lighter axon atrophy, regularly arranged nerve fibers, minor myelin sheath loss, and partial nerve fiber swelling; the dorsal root ganglion neurons were arranged loosely, and the intercellular structure was partially loose (Figure 3D).

It was thus found that alcoholism altered the histomorphology of the rat sciatic nerve, which was restored by the intervention of BMSCs and bFGF in the alcoholic rats.
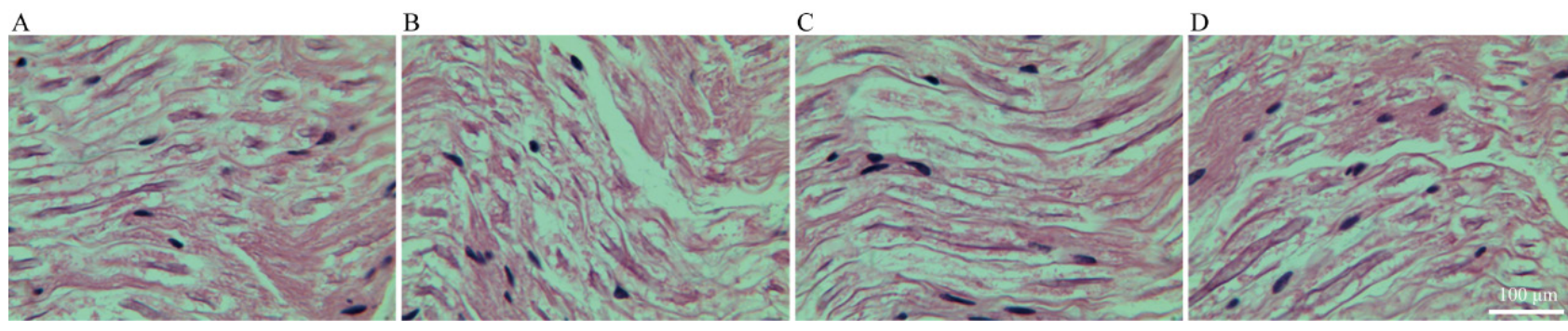

Figure 3 - Observation of sciatic nerve morphology in different groups.

\section{- Discussion}

The results showed that the serum SOD and MT contents in group BMSC were greater and the MDA content less compared to those in groups MOD and bFGF, the differences being statistically significant $(p<0.05)$.

BMSCs play a role in improving the metabolic capacity of free radicals in the CAI rat model. During long-term drinking, the microsomal enzyme oxidizing system (MEOS) is involved in ethanol oxidation by the microsomal enzymes, increasing the oxygen consumption by $50 \%$ to $60 \%$. The cells are in a state of high metabolism during alcohol metabolism and produce a large number of oxygen free radicals, thus resulting in lipid peroxidation ${ }^{17}$. MDA is an important product of lipid peroxidation 
in the body, and its content can be used as an important indicator of oxygen free radical damage to cells. Recent studies have shown that MT, as an effective free radical scavenger, has an important protective effect on tissues and cells under oxidative stress. It can directly capture a variety of reactive oxygen species and reactive nitrogen free radicals, scavenge radicals ${ }^{18}$, and play a neuroprotective role ${ }^{19}$. The decrease of MT content in group MOD indicated that the ability of the heart and brain blood vessels to scavenge free radicals was reduced. The increase of MT content in group BMSC indicated that BMSCs can increase the MT content in the rat CAI model.

Light microscopy of the sciatic nerve revealed that the sciatic nerve fibers in group BMSC were mostly arranged in a regular manner, and the morphology of the sciatic nerve tissues was recovered to a certain degree, indicating that the BMSC intervention could repair the sciatic nerve tissue damage. The results of stress relaxation and creep tests of the sciatic nerve in the different groups showed that at 7200s, stress loss and strain increase for the sciatic nerve in group BMSC were greater than those in groups MOD and bFGF, indicating that BMSC intervention in CAI rats can restore the stress relaxation and creep characteristics.

It was found that the changes in serum SOD, MT and MDA contents were related to the stress relaxation and creep properties of the sciatic nerve in rats. The contents of SOD and MT in serum decreased and that of MDA increased, and both decreased the stress relaxation and creep of the sciatic nerve in 7200s. It has been found that alcoholism induces ischemia and hypoxia in heart and cerebrovascular response in rats, which led to the release of free radicals in tissues, decreased the contents of SOD and MT and increased that of MDA, thus decreasing stress relaxation and the creep at 7200s in the sciatic nerve. After the intervention of BMSCs, the SOD content increased and MDA content decreased, which implied that the BMSCs helped to scavenge the free radicals in the alcoholic rats and protected the sciatic nerve, with the stress relaxation and creep properties recovered.

Barak et al. ${ }^{20}$ found that long-term alcohol intake can affect the proliferation and differentiation of neural stem cells; meanwhile, the astrocytes are very sensitive to the alcohol metabolite, acetaldehyde, and glial cell injury can damage the nutritional and metabolic regulation of the neurons, thus further accelerating development of neurodegenerative diseases. It is believed that long-term alcohol intake in CAI rats causes degenerative lesions in the sciatic nerve and reduces the proliferation of neural stem cells. Therefore, the stress relaxation and creep characteristics may change. The sciatic nerve fibrous tissue and neural stem cell injuries in groups BMSC and bFGF were repaired, so the stress relaxation and creep properties of the sciatic nerve were considered improved.

The results of this study showed that after the BMSC intervention, the serum biological parameters, sciatic nerve tissue morphology, and viscoelastic properties in group MOD were restored and improved, indicating that BMSCs can promote the regeneration of the sciatic nerve in CAl rats, which is consistent with the desired results.

The results of this study are in agreement with those of another study ${ }^{21}$, in which the transplantation of BMSCs helped in the recovery of damaged collagen fibers to a certain extent and thus improved the tensile biomechanical properties of the sciatic nerve. This study showed that BMSC transplantation improves not only the tensile biomechanical properties of the sciatic nerve but also the stress relaxation and creep properties of the sciatic nerve.

This study investigated the correlation 
of the stress/strain of the sciatic nerve with time and concluded that the correlation of the sciatic nerve strain with time reflects the viscoelastic characteristics of the sciatic nerve better. Therefore, it can provide a theoretical basis for the pathogenesis of CAl-induced sciatic nerve injury from the aspect of viscoelastic mechanics.

Owing to the limited sample size and the differences among the experimental animals, the experimental data obtained in this study have a certain degree of dispersion, but they still can be used for development of strategies for the prevention and treatment of CAI.

\section{Conclusion}

Bone mesenchymal stem cells can improve the metabolism of free radicals, restore the tissue morphology and viscoelasticity of the chronic alcohol intoxication animal model, and positively affect the repairing of the injured sciatic nerve.

\section{References}

1. Navarro Al, Mandyam CD. Protracted abstinence from chronic ethanol exposure alters the structure of neurons and expression of oligodendrocytes and myelin in the medial prefrontal cortex. Neuroscience. 2015;293:35-44. doi: 10.1016/j.neuroscience.2015.02.043.

2. Zhang $N$, Hu Y, Ding C, Zeng $W$, Shan $W$, Fan $H$, Zhao Y, Shi X, Gao L, Xu T, Wang R, Gao $D$, Yao J. Salvianolic acid B protects against chronic alcoholic liver injury via SIRT1mediated inhibition of CRP and ChREBP in rats. Toxicol Lett. 2017;267:1-10. doi: 10.1016/j.toxlet.2016.12.010.

3. Reinhart V, Bove SE, Volfson D, Lewis DA, Kleiman RJ, Lanz TA. Evaluation of TrkB and BDNF transcripts in prefrontal cortex, hippocampus, and striatum from subjects with schizophrenia, bipolar disorder, and major depressive disorder. Neurobiol Dis. 2015;77:220-7. doi: 10.1016/j. nbd.2015.03.011.

4. Victor M. Polyneuropathy due to nutritional deficiency and alcoholism. In: Dyck PJ, Thomas PK, Lambert E (Eds). Peripheral neuropathy. London: Saunders; 1975. p.1030-60.

5. Dixit D, Endicott J, Burry L, Ramos L, Yeung SY, Devabhakthuni S, Chan C, Tobia A, Bulloch MN. Management of acute alcohol withdrawal syndrome in critically III patients. Pharmacotherapy. 2016;36(7):797-822. doi: 10.1002/phar.1770.

6. Donnelly A. Wernicke-Korsakoff syndrome: recognition and treatment. Nurs Stand. 2017;31(31):46-53. doi: 10.7748/ns.2017. e10440.

7. Monforte R, Estruch R, Valls-Solé J, Nicolás J, Villalta J, Urbano-Marquez A. Autonomic and peripheral neuropathies in patients with chronic alcoholism. A dose-related toxic effect of alcohol. Arch Neurol. 1995;52(1):45-51. PMID: 7826275.

8. Tan ET, Johnson RH, Lambie DG, Whiteside EA. Alcoholic vagal neuropathy: recovery following prolonged abstinence. J Neurol Neurosurg Psychiatry. 1984;47(12):1335-7. PMID: 6512554.

9. Dezawa M, Takahashi I, Esaki M, Takano $M$, Sawada H. Sciatic nerve regeneration in rats induced by transplantation of in vitro differentiated bone-marrow stromal cells. Eur J Neurosci. 2001;14(11):1771-6. PMID: 11860471.

10.Wang $P$, Zhang $Y$, Zhao J, Jiang $B$. Intramuscular injection of bone marrow mesenchymal stem cells with small gap neurorrhaphy for peripheral nerve repair. Neurosci Lett. 2015;585:119-25. doi: 10.1016/j.neulet.2014.11.039.

11.Aebischer P, Salessiotis AN, Winn SR. Basic fibroblast growth factor released from synthetic guidance channels facilitates peripheral nerve regeneration across long nerve gaps. J Neurosci Res. 1989;23(3):2829. doi: 10.1002/jnr.490230306.

12. Rodd ZA, Bell RL, Sable HJ, Murphy JM, McBride WJ. Recent advances in animal models of alcohol craving and relapse. Pharmacol Biochem Behav. 2004;79(3):43950. doi: 10.1016/j.pbb.2004.08.018.

13.Zhang ZJ, Li YJ, Liu XG, Huang FX, Liu TJ, Jiang DM, Lv XM, Luo M. Human umbilical cord blood stem cells and brainderived 
neurotrophic factor for optic nerve injury: a biomechanical evaluation. Neural Regen Res. 2015;10(7):1134-8. doi: 10.4103/16735374.160110.

14.Wang Y, Li ZW, Luo M, Li YJ, Zhang KQ. Biological conduits combining bone marrow mesenchymal stem cells and extracellular matrix to treat long-segment sciatic nerve defects. Neural Regen Res. 2015;10(6):96571. doi: 10.4103/1673-5374.158362.

15.Jin $H$, Yang Q, Ji F, Zhang YJ, Zhao Y, Luo M. Human amniotic epithelial cell transplantation for the repair of injured brachial plexus nerve: evaluation of nerve viscoelastic properties. Neural Regen Res. 2015;10(2):260-5. doi: 10.4103/16735374.152380 .

16.Pan Y, Zhang L, Zhang $\mathrm{X}$, Hu C, Liu R. Biological and biomechanical analysis of two types of mesenchymal stem cells for intervention in chemotherapy-induced ovarian dysfunction. Arch Gynecol Obstet. 2017;295(1):247-52. doi: 10.1007/s00404016-4224-5.

17.Gitnick G. Principles and practice of gastroenterology and hepatology. 2ed. USA: Appleton and Lange Conn; 1994.

18.Cai L. Suppression of nitrative damage by metallothionein in diabetic heart contributes to the prevention of cardiomyopathy. Free Radic Biol Med. 2006;41(6):851-61. doi: 10.1016/j.freeradbiomed.2006.06.007.

19. Fink RC, Scandalios JG. Molecular evolution and structurefunction relationships of the superoxide dismutase gene families inAngiosperms and their relationship to other eukaryotic and prokaryotic superoxide dismutase. Arch Biochem Biophys. 2002;399(1):19-36. doi: 10.1006/ abbi.2001.2739.

20.Barak S, Wang J, Ahmadiantehrani S, Ben Hamida S, Kells AP, Forsayeth J, Bankiewicz KS, Ron D. Glial cell line-derived neurotrophic factor (GDNF) is an endogenous protector in the mesolimbic system against excessive alcohol consumption and relapse. Addict Biol. 2015;20(4):629-42. doi: 10.1111/ adb.12152.

21.Li YJ, Zhao BL, Lv HZ, Qin ZG, Luo M. Acellular allogeneic nerve grafting combined with bone marrow mesenchymal stem cell transplantation for the repair of longsegment sciatic nerve defects: biomechanics and validation of mathematical models. Neural Regen Res. 2016;11(8):1322-6. doi: 10.4103/1673-5374.189198.

\section{Correspondence:}

Daliang Kong,

Department of Orthopaedics

China-Japan Union Hospital, Jilin University

No. 126 Xiantai St,

Changchun 130031 China

Phone: +8643185693301

daliangkongcn@163.com

Received: June 10, 2018

Review: Aug 12, 2018

Accepted: Sept 10, 2018
Conflict of interest: none

Financial source: Jilin Provincial Science and

Technology Development Plans (20110492) 